\title{
The twelve colorful stones as building blocks
}

\section{R. M. Doria ${ }^{* \dagger}$}

AprendaNet - Petrópolis - RJ, Brazil

E-mail: doriadaprendanet.com.br

\section{J. A. Helayël}

CBPF - Centro Brasileiro de Pesquisas Físicas

E-mail: helayel@pq. cnpq.br

Based on twelve colourful stones and adopting $S U(3)$ as symmetry group, we build up candidates for new types of matter. Quarks and leptons are generated as the most fundamental composite structures. A first consistency of this model is that no other kind of particle is generated. It only says that, based on colour and spin, different new types of fermionic and bosonic quarks and leptons can be produced. It yields a new particle zoo to be considered at LHC, where, instead of being just a conserved charge, colour is proposed as one of nature substances.

7th International Conference on Mathematical Methods in Physics,

April 15 - 192012

Rio de Janeiro, Brazil

\footnotetext{
* Speaker.

${ }^{\dagger}$ Poster Section
} 


\section{Introduction}

Colour interpretation is being a challenge for physics. Formally, it is delivered as a conserved charge, but it has the strange property of being confined. What is colour? Our seek is that it should be understood as one of the substances that build up the elementary particles. Similarly to mass, colour should be worked out as nature primordial substance.

Thus, there is a colour region which is prior to the quarks one. This is our investigation. Colour and gauge symmetry develop the most subtle dynamics in nature. It should be identified as the dynamics of colourful stones []. Thus, before delivering QCD and its properties as confinement, one should first investigate on such colourful world by understanding its composition.

The first colourful challenge is to understand such new particles just made by colour and with a dynamics stipulated by gauge symmetry which will be called as colourful stones. What is their nature? How they build quarks and leptons? How many are they?

The fascinating aspect of the present-day physics is that, to understand these colourful stones, one has to look for clues. Being confined, the colour observation becomes indirected what gives for these colourful stones an intrinsic character. Different from the usual particles they cannot be measured. The only result for proposing these particles is that quarks contain three colours. And so from, this single tip one has to derive an entire colourful world.

Reducing to colourful stones is perhaps the best method for understanding quarks. It puts the question just on the meaning of colour. Through quarks usually colour is mixed with mass, charge and spin what does not clarify on its meaning. Our concept here is that colour should be recognized as the most subtle substance in nature. It says that quarks and leptons should be constituted by colour, and so, colour can be understood as the primordial substance.

Thus we are inside of a big challenge which is not to prove confinement but how from this colourful world one can build up another particles with colour and colourless. How? Given that experimentally the Universe was noticed as derived in extrinsic (measurable) and intrinsic (confined) structures, our intuition is that these two sides should share a common structure. So, under this suggestion, our guess is that similarly to the extrinsic Universe that is divided in fermions and bosons, its intrinsic side should follow the same nature. It is a spin clue, because it says that such colourful stones should also be divided into two families of bosons and fermions, that just for changing the terminology, we prefer to call them as YANG and YIN families. Perhaps, it is a more appropriate name for evolving this subtle colourful world.

The general idea here is to start nature process basing only on colour. In the Big Bang conception, the Universe has an alpha point determined by temperature parameter. It tells that the Universe after a high temperature state starts cooling. Our approach would be that, after a state that depends only on colour, it moves by creating singlets. The belief is that the Universe dynamics is carried out by a tendency for generating singlets that will be in continuous transformations. This evolution will represent rearrangements of the colourful structures. The tables build up in the text show a part of these possible colour and singlet states.

In this work, we are proposing a model for the initial colourful state. It is called the twelve colourful stones []. It is based on the existence of three colours into two families. The intuition for such a choice is based on two facts. For the first, observe the measurements of the total cross section of the annihilation of electron-positron pairs in colliding beam experiments []. The data 
up to C. M. energy $35.8 \mathrm{GeV}$ are an indication for three colours. Thus, we have called stones the particles whose dynamics is based only on the concepts of colour. For the second, the nature of these stones, observe now the measurable nature. It reveals two basic entities: the fermionic and the bosonic. We consider these two structures aspect as a second fact to guide us to investigate the colourful world also with two families. They were called by yang and yin. The yang structure is correlated with a spin zero field, $\phi^{i}$, and the yin with with a spin half field, $\psi^{j}$. As each family has three colours with the respective anticolour, we have twelve colourful stones. Physically, it will be important to mix these families. In order to do this it is necessary to define them with the same three colors. Then there is just one group involved. Therefore, the singlets can be build up either by elements of the same family or mixing them. The group considered is $S U(3)_{C}$.

In Parts 2 and 3, the states are systematically described by composition of stones. In Part 4, quarks and leptons are generated with bosonic and fermionic possibilities. In Part 5, the conclusion is presented asking for a unity between the yang and yin stones.

\section{Colourful stones as basics triplets}

Consider the yin case. The vector components $\psi_{i}$ for the fundamental representation 3 are the colourful states called yin stones those for the conjugate representation $\overline{3}$ are called anti-yin stones. More explicitly,

$$
\psi=\left(\begin{array}{l}
\psi_{1} \\
\psi_{2} \\
\psi_{3}
\end{array}\right) \quad \bar{\psi}=\left(\begin{array}{l}
\psi^{1} \\
\psi^{2} \\
\psi^{3}
\end{array}\right)
$$

with the rotation

$$
\psi^{\prime}=U \psi
$$

where

$$
U=e^{i \omega^{a} t_{a}}, \quad a=1, \ldots, 8
$$

Similarly for the yang case. The notation is as in Fig. 1. III is the fundamental representation for yang stones. The representations of $S U(3)_{C}$ can be build up by taking tensor products of independent representations as $3 \otimes \overline{3}, I I I \otimes \overline{I I I}, I I I \otimes \overline{3}$, etc. The irreducible representation is realized in the space of the tensor $T_{\alpha_{i} \ldots \alpha_{p}}^{\beta_{1} \ldots \beta_{q}}$, whose independent components may be regarded as a multiplet of dimension $D(p, q)$. These particles will be composed of stones (yang-yin) and/or anti-stones (yang-yin).
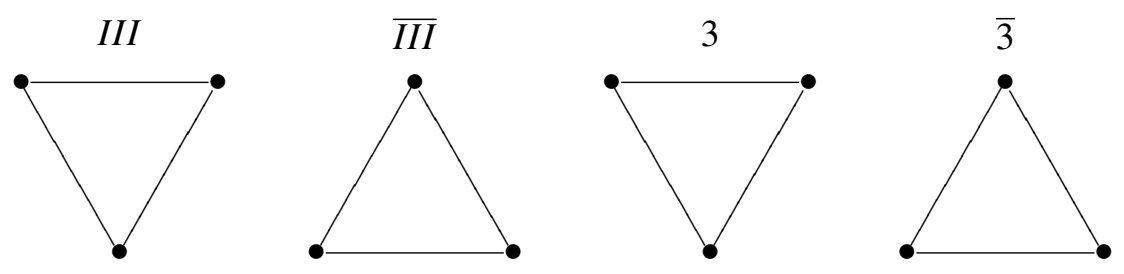

Figure 1: Yang-yin triplets are characterized by the roman and arabic numbers respectively. 
In addition to $S U(3)_{C}$, the lagrangian density [1] is also invariant under independent phase changes of $\phi$ and $\psi$. The transformation form a group $U(1) \times U(1)$. We can identify the generator of one of the $U(1)$ group as yang number $Y$, and call the generator of the other $U(1)$ group as yin number $y$. Thus, each state built up by the stones will be characterized by a $Y / y$ number. Therefore two colourful stone numbers can be assigned to all particles. The antiparticle is defined with opposite numbers. The conservation law is that $Y$ and $y$ are separately conserved. In Appendix A, we present an example.

\section{The Product of Representations}

Basing on the fundamental representations $3, \overline{3}, I I I, \overline{I I I}$, as in Fig. 1, we are going to study the multiplets. The structures to be generated will be less fundamental as the number of factors in the representation product increases. Essentially, the possibilities will be either to generate colourful or colourless structures. The stones have three ways to be arranged. They are the yang-yang, yin-yin and yang-yin compositions. Each $Y / y$ state has three kinds of partners. They are the opposite, complementary and reflexive states. The first is defined by having opposite yang and yin numbers. In the second, either the yang or the yin is opposite. In the last, these numbers are reflected. The states containing the same number of yang and yin stones will be arranged in sets. Each set is build up by basic states. This means that all other states of that set will be just the opposite and the complements of these basic states.

Consider a case with $n$ stones. The number of states generated by the yang or yin composition is $(n+1)$. There are $(n+1)$ sets. For an $n$ even, the number of bosonic sets is $\frac{n}{2}+1$. To the odd case is $\frac{n+1}{2}$. Suppose a set in the yang yin composition with $m$ yin stones. The number of states will be $(n-m+1)(m+1)$. When the number of both yin and yang is even, there will be $\left(\frac{m}{2}+1\right)\left(\frac{n-m}{2}+1\right)$ basic states in a set. If $m$ is odd and $n-m$ is even, will be $\left(\frac{n-m}{2}+1\right)\left(\frac{m+1}{2}\right)$. Similarly, for the other case.

The hadrons have been described by three quarks. Considering this, we are going to make a study up to six stones. In order to summarize the text, Tables up to three stones arrangement will be included.

\subsection{The composition of stones}

Observing first the cases where the fields $\phi_{i}$ and $\psi_{j}$ are mixed, we have

$$
\chi_{i}=f_{i}^{j k} \phi_{j} \psi_{k}
$$

and

$$
\eta=\phi_{i} \psi^{i}
$$

Equation (3.1) defines the quark. The colours of the fields are arranged in terms of the constants $f_{i j k}$. The form of this constant will depend on the irreducible representation where the quarks are defined. Equation (3.2) defines a lepton. Observe that there does not exist other possibility to mix the two fields different from (3.1) and (3.2). Thus, from the twelve colourful stones building blocks, it is not possible to generate a third kind of elementary structure with spin half. 
(3.1) and (3.2) in Table I are in the structures (g) and (h). There also appear the respective antiparticles through (i) and (j). However, from (a)-(f), there are other candidates to the fundamental particles. Such structures are bosonic. The expressions will be similar to (3.1) and (3.2) but with equal fields. They would produce respectively four and two kinds (one colourful and another colourless) of bosonic elementary particles.

\subsection{Three-Stone Composition}

Let us study it as a particular case of the $n$ stones generalization. The yang or yin composition has four states. There are four sets, where two are bosonic. Consider the set III on the Table II. It has just one yin stone. Thus, the number of states will be six. The number of basic states in this set is two. They are $1 / 2$ and $1 / 10$.

In total, Table II generates twenty states. It contains eight types of singlets. (a), (d), (o), (t) are bosons. (e), (h), (i), (n) are fermions. The yang yin crossing generates two sets. One with spin half and the other with spin zero or one.

\subsection{Four-Stone Composition}

There are five sets. The yang or yin composition has five states. Consider the set with two yin stones. It has nine states. The number of basic states in it is four. They are 2/2, 2/0, 0/2 and 0/0. The other states are complements. In total, there are eight colourless states. Each one contains two singlets. Therefore, we have as potentially experimental facts, eight fermionic structures and eight bosonic.

\subsection{Five-Stone Composition}

It has fifty six states. Six from yang composition, six from yin. It contains twenty kinds of colourless states. Each one has three types of singlets. We could associate in the yin composition, each singlet with each type of spin.

\subsection{Six-Stone Composition}

It has seventy one states, where twenty four are colourless. There are two kinds of colourless states. One has five singlets and the other six. The first has six bosonic and four fermionic structures. The other number of this structures are eight and six respectively. In total, the number of measurable fermions is different from the boson.

\section{Quarks and Leptons}

As quarks, we define the simplest colourful structure, irrespective of the spin. They can be either the triplets or the sextet in Table I. Quarks contents of 3, $\overline{3}, 6$ and $\overline{6}$ are in Figs. 2 and 3. Each figures will represent a meaning in the moment that the $Y / y$ quantum numbers are associate to them. They are 2/0,1/1,0/2 and the respective antiparticles. The fermionic quark field is

$$
\chi_{i}=f_{i}^{[j k]} \phi_{[j} \psi_{k]}
$$


where $i=1,2,3$. This means three quarks with different colours. The constant $f_{i}^{[j k]}$ is the antisymmetric tensor $\varepsilon_{i j k}$. It yields,

$$
\bar{\chi}^{i} \chi_{i}=\phi_{j}^{*} \phi^{j} \bar{\psi}_{k} \psi^{k}-\phi_{l}^{*} \phi^{j} \bar{\psi}_{j} \psi^{l}
$$

(4.2) is gauge invariant under (2.2).
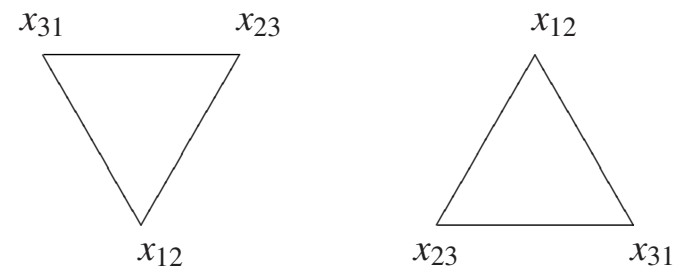

Figure 2: Quarks as members of a triplet with a corresponding $Y / y$ number. $\chi_{i}$ represents a colourful composite field. It appears three different natures for quarks.

For the sextet case,

$$
\chi_{i}=g_{i}^{(j k)} \phi_{(j} \psi_{k)}
$$

where $i=1, \ldots, 6$. The symmetric $g_{i(j k)}$ satisfy the relation

$$
g_{i(j k)} g_{i(l m)}=\delta_{j l} \delta_{k m}+\delta_{j m} \delta_{k l} .
$$

It yields

$$
\bar{\chi}_{i} \chi^{i}=\varphi_{j}^{*} \varphi^{j} \bar{\chi}_{k} \chi^{k}+\varphi_{k}^{*} \varphi_{j} \bar{\chi}^{j} \chi^{k}
$$

Similarly to (4.2) the composition in the sextet will be invariant. In Appendix B the generators for the sextet are calculated.
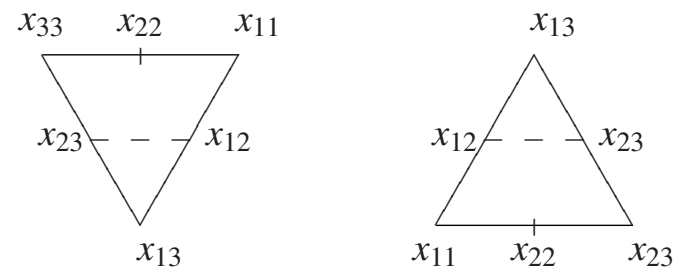

Figure 3: Quarks as sextet members.

Following the eightfold way [3], we define

$$
Q^{c}=I_{3}^{c}+\frac{y^{c}}{2}
$$

with the difference that $I_{3}^{c}$ and $y^{c}$ are colour dependent. They can not be interpreted with observables directly. In this way a colourful state is defined by a $Y / y$ number and $Q^{c}$. For instance, the spin half quark has nine states. Adding to it the bosonic structures there are ninety possible states.

We interpret the lepton as the singlets in Table I. Because stability the electron is chosen as the most basic lepton. Therefore, we have to define it from the Table I. It would be the state 1/-1. Then, looking at Table I, the positron and two bosonic partners would appear. 


\section{Conclusion}

Confinement is being a hard theme for physics. Since 1967 that our accelerators have detected quarks only indirectly. After 30 years of research no free quark has appeared. Our viewpoint is that the keyword to be understood is colour. It is the entity that makes a quark different from an electron. So, what is colour?

Colour interpretation is a challenge for physics. Our context is that it should be treated as a substance. We would say it is the most primeval nature entity. Our approach is that colour should be a substance with the following properties: generate particles and with the properties of asymptotic freedom and confinement.

In our days, before LHC experiments, quarks and leptons are presented as indivisibles. Thus such twelve colourful stones are introduced as a model to create a common origin for quarks and leptons based on colour and introducing a new aspect for minimum block called colourful stone. It is a model able to predict particles in a colourful side and particles in a singlet side of nature. Thus a first step to understand colour was established. Tables I and II are showing how from twelve colourful stones prescription one is able to cross from one side to another one.

Since long ago, it is being required the presence of an indivisible structure. In some senses, depending on accelerator generation, physics will be offering some candidates to building block. The present experimental spectrum suggests quarks and leptons as non-composite particles. However, they have distinct properties. Thus, it becomes important to clarify what are the conditions for elementarity.

With the advent of quark physics, the concept of colour has started being revealed. The question is whether it is an entity just correlated with quarks or a general element of nature. The meaning of the tables in text is that all particles would have an origin in colour. We have presented a study based on $S U(3)_{C}$ group. These tables constitute the engineering for building up structures flowing in the colourful river. These states have opposite, complements and reflexive forms. They put us in contact with physics in the moment that the candidates to observable states, the colour singlets can systematically be generated. The basis of them is the presence of two distinct families (spin half and spin zero) transforming under the fundamental representation of the same group.

The use of a symmetric group here was done in an opposite path to the eight fold way. There, starting from observables as isotopic spin and hypercharge, we were led to an $S U$ (3) flavour group. Here, the physical states are not intended to be classified into multiplets, but rather as singlets of the symmetric group. It is left as a posteriori the identification of these states.

Our effort at this work was just to treat colour as the substance maker of quarks and leptons. While mass is a substance that can be treated through the inertia principle, colour should be studied under a symmetry principle. Based just in $S U(3)_{C}$, one is led to the presence of twelve colourful stones as the most fundamental building block in nature. It derives just quarks (triplets and sextets) and leptons (singlets), as eq. (3.1) and (3.2) are showing.

We would observe that these twelve colourful stones approach is being based only on an internal colour group. It brought a new way to build up the bosonic and fermionic colourless world. The origin of them is in two kinds (yang and yin) of building blocks. However, this fact has not been deeply exploited in the lack of a connection between the colour and the space time structure. In the moment this connection is established we will have achieved a full unification in the colourful 
worl. This naturally motivates the introduction of supersymmetry. The emerged physical states were up to now unconnected. This colour unity will bring a correlation between these observable states.

We would also like to observe that the appearance of a sextet of quarks, as discussed in Section 4 , is a nice feature of the model proposed here. More recently, quarks sextets have been considered in connection with the lattice aproach to QCD []. Also, pentaquarks [], another relevant topic in connection with hadron physics may naturally arise from our model: $\underline{6} \otimes \underline{6} \otimes \underline{6} \otimes \underline{6} \otimes \underline{6}^{*}$ and $\underline{3} \otimes \underline{3} \otimes \underline{3} \otimes \underline{6} \otimes \underline{6}^{*}$. Indeed, these tensor products with sextet quarks yield colour singlets made up of 5 quarks. This aspect, and an appropriate gauge model to describe pentaquarks, requires special attention and we shall soon report on it elsewhere. The interesting outcome is that, with colour sextets, pentaquarks are not forbidden. There still remains a non-trivial and very relevant problem: the generation of a mass scale for the colour sextets and the estimation of how heavier than the triplet quarks they are. We must seek a mechanism to generate mass hierarchies from our composite model.

As a second aspect for understanding colour substance it is to relate it with the asymptotic freedom property. This means to propose a dynamics under a Lagrangian containing three and four-gauge-boson vertices. Based on eq. (2.1) this Lagrangian is being under study [].

A third colour property is a Gauss law with linear potentials. This should be taken as the classical prescription for justifying colour confinement. Again in [] one expects from this new $S U(3)_{C}$ Lagrangian something different from QCD. It is a challenge for next papers including massive gluons.

Thus we would summarize the twelve colourful stones model with the following aspects to be understood:

(i) The only fundamental parameter is colour. It remains to be shown in future a dependance of mass and charge with colour.

(ii) The building block is the so called yang-yin structure. From it, we can generate quarks and leptons according to section 4 . Thus, a further yang-yin unity is required. Supersymmetry can help.

(iii) The candidate gauge invariant Lagrangian for the gauge fields intermediating these colourful stones have the three-gauge-boson-vertex. Therefore, we expect the property of asymptotic freedom.

(iv) Colour confinement should be investigated through colourful stones prior than quarks.

There is something beyond QCD to be tested at LHC. Based on twelve colourful stones there is a model for understanding colour. Besides composite quarks and leptons, new particles and $S U(3)_{C}$ Lagrangian it says that together with the phenomenological region nature contains a noumenonic region based on colour to be understood. There it is the origin of the usual physical matter.

\section{Appendix A}

Considering that $\phi$ and $\psi$ are independent fields the yang and yin numbers conserve separately. This defines a particle with the structure

$$
\text { Particle } \equiv \text { Yang Number / Yin Number }
$$


Under this assigment, the simplest compositions were studied in Table I. They were identified as quarks and leptons. Choosing the electrons as basis, define

$$
e^{-} \equiv 1 /-1, \quad e^{+} \equiv-1 / 1
$$

Stable particles as $v, \mu, \tau$ can not have their $\mathrm{Y} / \mathrm{y}$ number determined from the electron case. Thus in principle they will be defined as

$$
v \equiv x /-x
$$

Let us analyse the reaction

$$
e^{+}+e^{-} \rightarrow q^{+}+q^{-}
$$

under Y/y number,

$$
-1 / 1+1 /-1 \rightarrow 1 / 1+-1 /-1
$$

(A.5) trivially realizes the prescription that the yang yin numbers conserve independently.

\section{Appendix B: Generators in the Sixth Representation}

The quarks generated from the twelve colourful stones can be triplets and sextets multiplets. Thus becomes necessary to calculate the generators for the 6 and $\overline{6}$ representations. The states are parametrized as in Fig. B1. The I, U and U spines are defined as in the Gell-Mann matrices. Defining,

$$
\begin{gathered}
\mathrm{I} \psi_{1}=\sqrt{2} \psi_{2} \quad \begin{array}{rl}
\mathrm{I} \psi_{4}=0 \\
\mathrm{I} \psi_{2}=\sqrt{2} \psi_{3} & \mathrm{I} \psi_{5}=0 \\
\mathrm{I} \psi_{3}=0 & \mathrm{I} \psi_{6}=\psi_{4}
\end{array} \\
\lambda_{1}=\frac{1}{2}\left(\begin{array}{ccccccc}
0 & \sqrt{2} & 0 & 0 & 0 & 0 \\
\sqrt{2} & 0 & \sqrt{2} & 0 & 0 & 0 \\
0 & \sqrt{2} & 0 & 0 & 0 & 0 \\
0 & 0 & 0 & 0 & 0 & 1 \\
0 & 0 & 0 & 0 & 0 & 0 \\
0 & 0 & 0 & 1 & 0 & 0
\end{array}\right)
\end{gathered}
$$






Fig. B1. Quarks in the sixth representation.

$$
\begin{gathered}
\lambda_{2}=-\frac{i}{2}\left(\begin{array}{cccccc}
0 & \sqrt{2} & 0 & 0 & 0 & 0 \\
-\sqrt{2} & 0 & \sqrt{2} & 0 & 0 & 0 \\
0 & -\sqrt{2} & 0 & 0 & 0 & 0 \\
0 & 0 & 0 & 0 & 0 & -1 \\
0 & 0 & 0 & 0 & 0 & 0 \\
0 & 0 & 0 & 1 & 0 & 0
\end{array}\right) \\
\lambda_{3}=\left(\begin{array}{cccccc}
1 & 0 & 0 & 0 & 0 & 0 \\
0 & 0 & 0 & 0 & 0 & 0 \\
0 & 0 & -1 & 0 & 0 & 0 \\
0 & 0 & 0 & -\frac{1}{2} & 0 & 0 \\
0 & 0 & 0 & 0 & 0 & 0 \\
0 & 0 & 0 & 0 & 0 & \frac{1}{2}
\end{array}\right) \\
\lambda_{4}=\frac{1}{2}\left(\begin{array}{llllll}
0 & 0 & 0 & 0 & 0 & 0 \\
0 & 0 & 0 & 0 & 0 & 1 \\
0 & 0 & 0 & \sqrt{2} & 0 & 0 \\
0 & 0 & \sqrt{2} & 0 & \sqrt{2} & 0 \\
0 & 0 & 0 & \sqrt{2} & 0 & 0 \\
0 & 1 & 0 & 0 & 0 & 0
\end{array}\right)
\end{gathered}
$$




$$
\begin{gathered}
\lambda_{5}=-\frac{i}{2}\left(\begin{array}{ccccccc}
0 & 0 & 0 & 0 & 0 & 0 \\
0 & 0 & 0 & 0 & 0 & -1 \\
0 & 0 & 0 & -\sqrt{2} & 0 & 0 \\
0 & 0 & \sqrt{2} & 0 & -\sqrt{2} & 0 \\
0 & 0 & 0 & \sqrt{2} & 0 & 0 \\
0 & 1 & 0 & 0 & 0 & 0
\end{array}\right) \\
\lambda_{6}=\frac{1}{2}\left(\begin{array}{ccccccc}
0 & 0 & 0 & 0 & 0 & \sqrt{2} \\
0 & 0 & 0 & 1 & 0 & 0 \\
0 & 0 & 0 & 0 & 0 & 0 \\
0 & 1 & 0 & 0 & \sqrt{2} & 0 \\
0 & 0 & 0 & \sqrt{2} & 0 & 0 \\
\sqrt{2} & 0 & 0 & 0 & 0 & 0
\end{array}\right) \\
\lambda_{7}=-\frac{i}{2}\left(\begin{array}{ccccccc}
0 & 0 & 0 & 0 & 0 & -\sqrt{2} \\
0 & 0 & 0 & -1 & 0 & 0 \\
0 & 0 & 0 & 0 & 0 & 0 \\
0 & 1 & 0 & 0 & -\sqrt{2} & 0 \\
0 & 0 & 0 & \sqrt{2} & 0 & 0 \\
\sqrt{2} & 0 & 0 & 0 & 0 & 0 & 0
\end{array}\right) \\
1 \\
\left.\begin{array}{llllllll}
-2 & 0 & 0 & 0 & 0 & 0 \\
0 & -2 & 0 & 0 & 0 & 0 \\
0 & 0 & -2 & 0 & 0 & 0 \\
0 & 0 & 0 & 1 & 0 & 0 \\
0 & 0 & 0 & 0 & 4 & 0 \\
0 & 0 & 0 & 0 & 0 & 1
\end{array}\right)
\end{gathered}
$$

The generators for $\overline{6}$ will be the complex conjugate of (B.2). 
$\left.T_{\alpha \gamma \beta}+T_{\beta \gamma \alpha}+T_{\gamma \beta \alpha}+T_{\gamma \alpha \beta}+T_{\beta \alpha \gamma}-T_{\beta \alpha \gamma}-T_{\beta \alpha \gamma}-T_{\alpha \gamma \beta}-T_{\gamma \alpha \beta}-T_{\gamma \beta \alpha}-T_{\alpha \beta \gamma}\right]$.

The decuplet is: $\frac{1}{2}\left[T_{\alpha \beta \gamma}+T_{\beta \gamma \alpha}+T_{\gamma \alpha \beta}+T_{\beta \alpha \gamma}+T_{\alpha \gamma \beta}+T_{\gamma \beta \alpha}\right]$.

(***) The triplet tensor is $\phi_{(\alpha \beta)} \phi^{\gamma} . \overline{6} \oplus 15$ gives $\phi_{[\alpha \beta]} \phi^{\gamma}$.

$(* * * *)$ It is as $(* * *)$ but changing upper by lower indices and vice versa.

$(* * * * *)$ Similarly to the case above.

(+) For each tensor change the field $\phi$ by $\psi$.

Yang Yin Crossing Yang/Yin Spin Tensor $\left(\right.$ Set III) ${ }^{(\dagger)} \quad$ Number

$\begin{array}{lllll}\text { (i) } & I I I \times I I I \times 3=\text { (a) } & 2 / 1 & 1 / 2 & \text { (S) } \\ \text { (j) } & I I I \times I I I \times \overline{3}=\text { (b) } & 2 /-1 & 1 / 2 & \text { (††) } \\ \text { (k) } & I I I \times \overline{I I I} \times 3=\text { (b) } & 0 / 1 & 1 / 2 & \text { (S) } \\ \text { (l) } & I I I \times \overline{I I I} \times \overline{3}=(\overline{\mathrm{b}}) & 0 /-1 & 1 / 2 & \text { (S) } \\ \text { (m) } & \overline{I I I} \times \overline{I I I} \times 3=(\overline{\mathrm{b}}) & -2 / 1 & 1 / 2 & \text { (S) } \\ \text { (n) } & \overline{I I I} \times \overline{I I I} \times \overline{3}=(\overline{\mathrm{a}}) & -2 /-1 & 1 / 2 & \text { (S) }\end{array}$

(S) It is similar to the case above.

$(\text { Set IV })^{(\circ)}$

$\begin{array}{lllll}\text { (o) } & I I I \times 3 \times 3=(\mathrm{a}) & 1 / 2 & 0,1 & (\mathrm{~S}) \\ \text { (p) } & I I I \times 3 \times \overline{3}=(\mathrm{b}) & 1 / 0 & 0,1 & (\circ \circ) \\ \text { (q) } & I I I \times \overline{3} \times \overline{3}=(\overline{\mathrm{b}}) & 1 /-2 & 0,1 & (\mathrm{~S}) \\ \text { (r) } & \overline{I I I} \times 3 \times 3=(\mathrm{b}) & -1 / 2 & 0,1 & (\mathrm{~S}) \\ \text { (s) } & \overline{I I I} \times 3 \times \overline{3}=(\overline{\mathrm{b}}) & -1 / 0 & 0,1 & (\mathrm{~S}) \\ \text { (t) } & \overline{I I I} \times \overline{3} \times \overline{3}=(\overline{\mathrm{a}}) & -1 /-2 & 0,1 & (\mathrm{~S})\end{array}$

(†) $T_{\alpha \beta \gamma}=\phi_{\alpha} \phi_{\beta} \psi_{\gamma}$

$(\dagger \dagger)$ The triplet tensor is $\phi_{(\alpha \beta)} \psi^{\gamma}$. Similarly, $\overline{6} \oplus 15$ gives $\phi_{[\alpha \beta]} \psi^{\gamma}$.

(о) $T_{\alpha \beta \gamma}=\psi_{\alpha} \psi_{\beta} \phi_{\gamma}$.

(००) The triplet tensor is $\psi_{(\alpha \beta)} \phi^{\gamma}$. Similarly, $\overline{6} \oplus 15$ gives $\psi_{[\alpha \beta]} \phi^{\gamma}$.

\section{References}

[1] R. M. Doria, The Twelve Colourful Stones - Brazilian Center of Physics Research CBPF preprint.

[2] Particle Data Group, Rev. Mod. Phys. 52 (1980) 556; D. W. Greenberg and C. A. Nelson, Physics Reports, 32C (1977) 71.

[3] Murray Gell-Mann Selected Papers, Worl Scientific Publishing (2010); M. Gell-Mann, Report CTSL-20 (1961), unpublished; Phys. Rev. 106 (1957) 1296

[4] Y. Ne'eman, Nucl. Phys. 26 (1961) 222.

[5] G. Zweig, CERN Reports TH. 401 and TH. 402 (1964).

[6] O. Machtey and B. Svetitsky, Metastable non confining states in SU(3) lattice gauge theory with sextet fermions, arXiv:0311.0886v2.

[7] B. Wybourne, Group Theory and Pentaquark, arXiv:hep-ph/0307170v2.

[8] R. Doria, Intrinsec Gauge Theory, submitted paper (2010); R. Doria and M.J. Neves, Proceedings of Science, PoS (ISFTG) 022 (2009). 